\author{
Supporting Information
}

\title{
Multifunctional Programmable DNA Nanotrain for Activatable Hypoxia Imaging and Mitochondria-targeted Enhanced Photodynamic Therapy
}

Jin Liu ${ }^{\mathrm{a}}$, Ge Ding ${ }^{\mathrm{b}}$, Shiya Chen ${ }^{\mathrm{a}}$, Caoye Xue ${ }^{\mathrm{c}}$, Mian Chen ${ }^{\mathrm{b}, *}, \mathrm{Xu}$ Wu ${ }^{\mathrm{d}, *}$, Quan Yuan , Jing Zheng, ${ }^{\mathrm{a}, *}$, Ronghua Yang ${ }^{\mathrm{e}}$

aState Key Laboratory of Chemo/Biosensing and Chemometrics, College of Chemistry and Chemical Engineering, Institute of Chemical Biology and Nanomedicine (ICBN), Hunan University, Changsha, 410082, China;

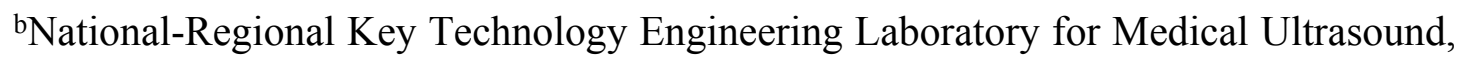
Guangdong Key Laboratory for Biomedical Measurements and Ultrasound Imaging, Health Science Center, School of Biomedical Engineering, Shenzhen University, Shenzhen 518055, China; 'Hunan Institute of Sports Science, Changsha, 410003, China; ${ }^{\mathrm{N} H C}$ Key Laboratory of Carcinogenesis and Key Laboratory of Carcinogenesis and Cancer Invasion of the Chinese Ministry of Education, Cancer Research Institute and School of Basic Medical Science, Central South University, Changsha, 410083, China; eSchool of Chemistry and Biological Engineering, Changsha University of Science and Technology, Changsha, 410004, China.

Corresponding authors' email address:

mian.chen@szu.edu.cn; wuxu1028@csu.edu.cn; zhengjing2013@hnu.edu.cn 


\section{Table of Contents}

1. Experimental Section

1.1 Materials and Apparatus

1.2 Stability of DNA nanotrain

1.3 Calculation of singlet oxygen quantum yield

1.4 Cell culture

1.5 Colocalization analysis

1.6 Mitochondrial membrane potential (MMP) analysis

1.7 Cell death assay

2. Oligonucleotides Sequences

3. Experimental Data

3.1 Characterization of nanotrain@TMPyP4

3.2 Stability study experiment

3.3 Fluorescence experiments in aqueous solution

3.4 Confocal fluorescence imaging in cells

3.5 Colocalization experiment and MTT assay

4. References 


\section{Experimental Section}

1.1 Materials and Apparatus. The sequences of the oligonucleotides used in this experiment are described in Table S1. Mito-Tracker Green was from KeyGEN BioTECH (Beijing, China). Singlet oxygen sensor green (SOSG) and propidium iodide (PI) were purchased from Invitrogen (Thermo Fisher Scientific, USA). 2',7'-dichlorofluoresceindiacetate (DCFH-DA) was from Maokang Biotech Co., Ltd. (Shanghai, China). Trypsin ethylenediaminetetraacetic acid, Dulbecco's modified Eagle's medium, penicillin, streptomycin and fetal bovine serum were acquired from Changsha Dingguo Biotechnology Co., Ltd. (Changsha, China). Fluorescence emission spectra were measured on a PTI QM4 fluorescence system (Photo Technology International, Birmingham, NJ) with accessories of a temperature controller. Fluorescence images of cells were obtained using an Olympus FV1000-MPE multiphoton laser scanning confocal microscope (Japan).

1.2 Stability of nanotrain. Evaluation of the stability of nanotrains in biological media using natural polyacrylamide gel electrophoresis (PAGE). The nanotrain was incubated with water, PBS and cell medium supplemented with $10 \%$ fetal bovine serum for $24 \mathrm{~h}$, respectively.

\subsection{Calculation of singlet oxygen quantum yield. Typically,} UB-nanotrain@TMPyP4 (equal to $1 \mu \mathrm{M}$ of TMPyP4) were respectively dissolved in PBS ( $\mathrm{pH}=7.4$ ) containing $2 \mu \mathrm{M}$ of SOSG. Then, the solutions were irradiated with a $660 \mathrm{~nm}$ light $\left(2.2 \mathrm{~mW} \cdot \mathrm{cm}^{-2}\right)$. The fluorescence emission was recorded at various irradiation times. The singlet oxygen $\left({ }^{1} \mathrm{O}_{2}\right)$ quantum yield $(\Phi)$ of the UB-nanotrain@TMPyP4 was calculated using the following equation ${ }^{1-3}$ :

$\Phi_{\text {UB-nanotrain@TMPyP4 }}=\Phi_{\text {TMPyP4 }} \times \mathrm{K}_{\mathrm{UB} \text {-nanotrain@TMPyP4 }} \times \mathrm{A}_{\text {TMPyP4 }} /\left(\mathrm{K}_{\mathrm{TMPyP} 4} \times\right.$ AUB-nanotrain@TMPyP4) 
where $\Phi_{\mathrm{UB}-\text { nanotrain@TMPyP4 }}$ is the ${ }^{1} \mathrm{O}_{2}$ quantum yield of the UB-nanotrain@TMPyP4, and $\Phi_{\mathrm{TMPyP}}$ is the ${ }^{1} \mathrm{O}_{2}$ quantum yield of TMPyP4. The value of $\Phi_{\mathrm{TMPyP}}$ is 0.77 in water. ${ }^{4} \mathrm{~K}$ represents the reaction rate constant of SOSG by UB-nanotrain@TMPyP4 ( $\left.\mathrm{K}_{\mathrm{UB}-\text { nanotrain@TMPyP4}}\right)$ or TMPyP4 ( $\left.\mathrm{K}_{\mathrm{TMPyP} 4}\right)$, and $\mathrm{A}$ represents the absorbance of TMPyP4 (A $\left.\mathrm{A}_{\text {TMPyP4}}\right)$ or UB-nanotrain@TMPyP4 (A $\left.\mathrm{A}_{\mathrm{UB}-n a n o t r a i n @ T M P y P 4}\right)$ at 660 nm.

1.4 Cell culture. MCF-7 cells were cultured in dulbecco's modified eagle medium (DMEM) supplemented with $10 \%$ fetal bovine serum (FBS) and $1 \%$ streptomycin-penicillin at $37^{\circ} \mathrm{C}$ in a humid atmosphere with $5 \% \mathrm{CO}_{2}$.

1.5 Colocalization analysis. MCF-7 cells were seeded in confocal dishes $\left(1 \times 10^{5}\right.$ cells per dish). After $24 \mathrm{~h}$ incubation at $37^{\circ} \mathrm{C}$, the media were replaced by $1 \mathrm{~mL}$ nanotrain $(50 \mathrm{nM})$ in the DMEM medium and incubated for $4 \mathrm{~h}$ under $1 \% \mathrm{O}_{2}$. After that, MCF-7 cells were incubated with fresh medium containing $100 \mathrm{nM}$ Mito-tracker green for $30 \mathrm{~min}$ for fluorescence imaging. The Pearson correlation coefficient was calculated by Image $\mathrm{J}$.

1.6 Mitochondrial membrane potential (MMP) analysis. We investigated the effect of nanotrain@TMPyP4 on MMP analysis using 5,5',6,6'-tetrachloro-1,1',3,3'-tetraethylbenzimidazolylcarbocyanine iodide (JC-1) dye-based fluorescent probe. As a lipophilic cationic dye, JC-1 easily enters and accumulates as aggregates in mitochondria which can emit red fluorescence. Once MMP decreases, the JC-1 dye outflows into cytoplasm and exists as monomers which can emit green fluorescence. Specifically, MCF-7 cells were seeded in confocal dishes $\left(1 \times 10^{5}\right.$ cells per dish) for $24 \mathrm{~h}$. After that, MCF-7 cells were incubated with nanotrain@TMPyP4 at $37^{\circ} \mathrm{C}$ for $4 \mathrm{~h}$ under $10 \% \mathrm{O}_{2}$. Then, cells were washed three times with PBS buffer and incubated with $10 \mu \mathrm{g} \cdot \mathrm{mL}^{-1} \mathrm{JC}-1$ at $37^{\circ} \mathrm{C}$ for $20 \mathrm{~min}$. The JC-1 dyes in cells were visualized by Olympus FV1000-MPE multiphoton laser 
scanning confocal microscope.

1.7 Cell death assay. MCF-7 cells were seeded in confocal dishes $\left(1 \times 10^{5}\right.$ cells per dish) for $24 \mathrm{~h}$. Then the cells were incubated with nanotrain@TMPyP4 for $4 \mathrm{~h}$ under different conditions. After that, the cells were irradiated by $660 \mathrm{~nm}$ light at a density of $2.2 \mathrm{~mW} \cdot \mathrm{cm}^{-2} .30 \mathrm{~min}$ later, the cell was stained with Hoechest and PI at room temperature for $10 \mathrm{~min}$ in the dark. The fluorescence intensity of cells was measured by Olympus FV1000-MPE multiphoton laser scanning confocal microscope with excitation wavelength of $488 \mathrm{~nm}$. The blue channel for Hoechest was excited at 405 $\mathrm{nm}$ and the fluorescence emission signal at 410-500 $\mathrm{nm}$ was collected. The red channel for PI was excited at $561 \mathrm{~nm}$ and the fluorescence emission signal at 590-640 nm was collected.

\section{Oligonucleotides Sequences}

\begin{tabular}{|c|c|}
\hline Name & Sequence (5'--3 ') \\
\hline $\begin{array}{l}\text { Initiation } \\
\text { probe }\end{array}$ & AAGGACCACCGCATCTCTA \\
\hline H1 & CACCGCATCTCTACCTAAGTGTAGAGATGCGGTGGTCCTTCCTCC \\
\hline $\mathrm{H} 2$ & $\begin{array}{l}\text { GGTGGTGGTGGTTGTGGTGGTGGTGGACTTAGGTAGAGATGCG } \\
\text { GTGAAGGACCACCGCATCTCTAC }\end{array}$ \\
\hline $\mathrm{H} 1-\mathrm{Cy} 3$ & $\begin{array}{l}\text { CACCGCATCTCTACCTAAGTGTAGA/iCy3/GATGCGGTGGTCCTTC } \\
\text { CTCC }\end{array}$ \\
\hline H2- BHQ2 & $\begin{array}{l}\text { GGTGGTGGTGGTTGTGGTGGTGGTGGACTTAGGTAGAGATGCG } \\
\text { GTGAAGGACCACCGCATCTCTAC - BHQ2 }\end{array}$ \\
\hline
\end{tabular}

* The G-quadruplex fragments are represented in underlined portions.

Table S1. Oligonucleotides used in this work. 


\section{Experimental Data}

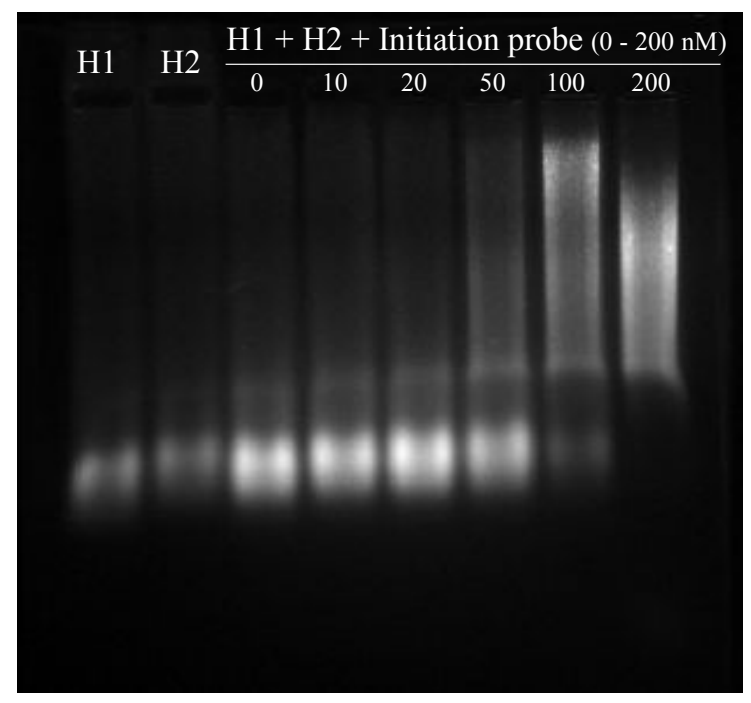

Figure S1. SYBR Green-stained 2\% agarose gel electrophores showing the effect of initiation probe concentration on the self-assemble of the nanotrain.
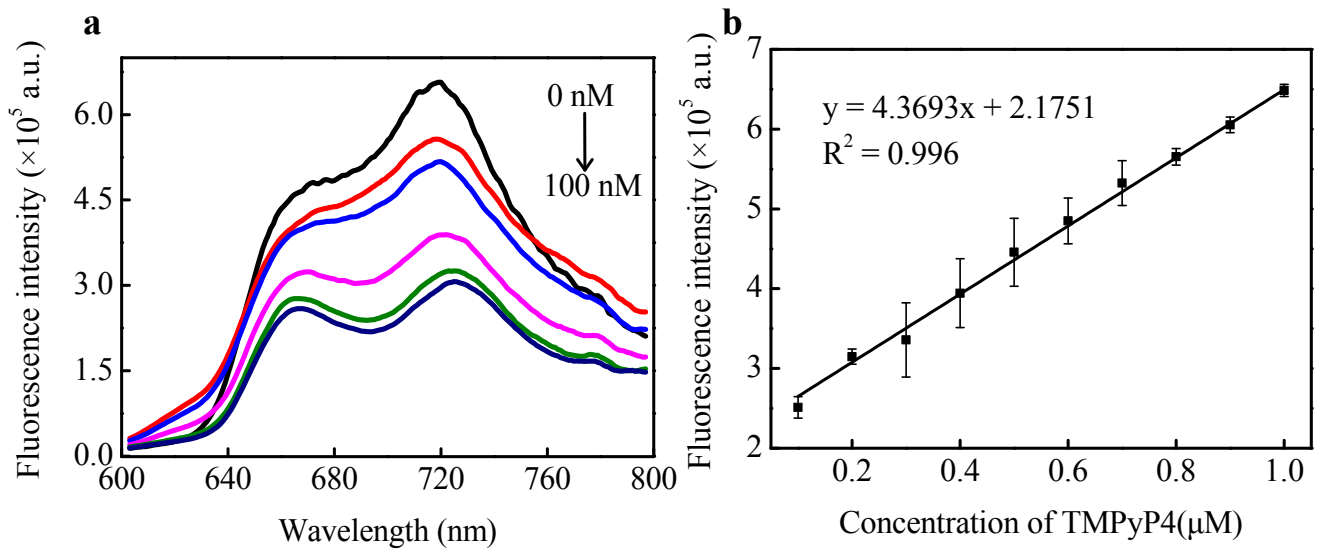

Figure S2. (a) Fluorescence emission spectra of TMPyP4 solution $(1 \mu \mathrm{M})$ with increasing concentrations of nanotrain (from top to bottom: 0, 10, 25, 50, 75 and 100 $\mathrm{nM}$ ) with an excitation wavelength of $435 \mathrm{~nm}$. (b) Standard curve based on fluorescence intensity of free TMPyP4 $(0.1-1 \mu \mathrm{M})$. Error bars represent standard deviations from three repeated experiments. 

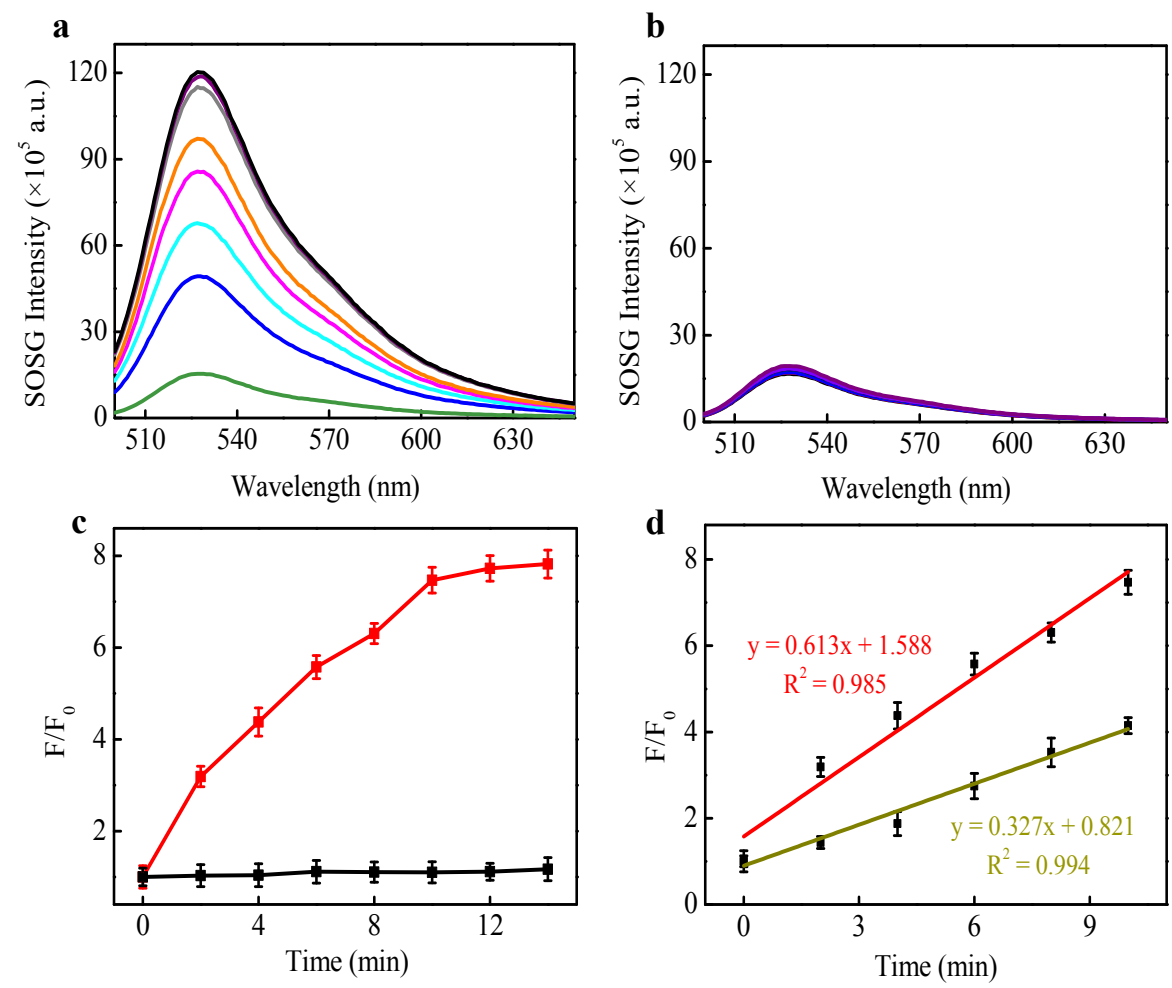

Figure S3. (a) Fluorescence emission spectra of UB-nanotrain@TMPyP4 upon different irradiation time under the excitation wavelength of SOSG. (b) Fluorescence emission spectra of nanotrain@TMPyP4 upon different irradiation time under the excitation wavelength of SOSG. (c) Fluorescence enhancement $\left(F / F_{0}\right.$, where $F$ and $F_{0}$ represents the fluorescence intensity of SOSG after and before light irradiation) of the nanotrain@TMPyP4 (black curve) and UB-nanotrain@TMPyP4 (red curve) upon different irradiation time. Error bars represent standard deviations from three repeated experiments. (d) Corresponding reaction rate constants of SOSG by TMPyP4 (dark yellow curve) and UB-nanotrain@TMPyP4 (red curve) under irradiation. 


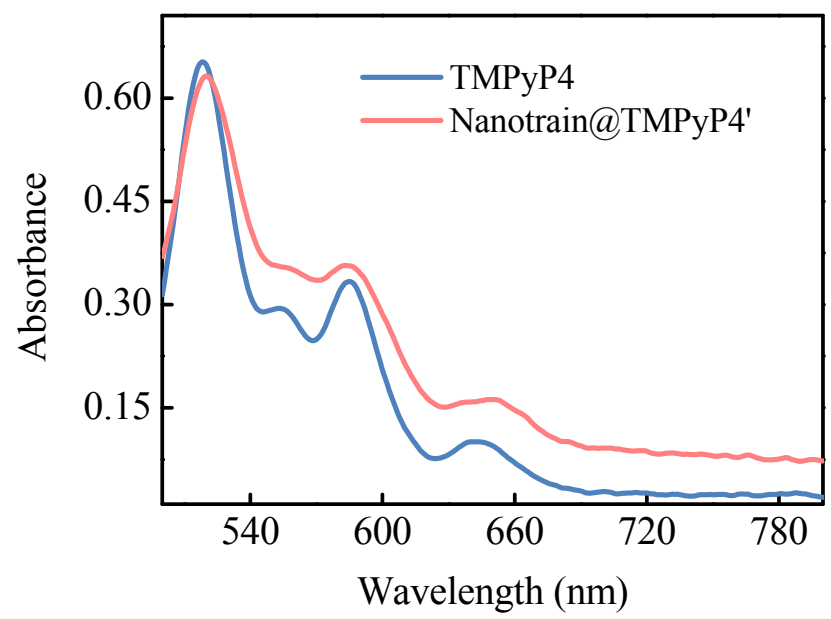

Figure S4. The UV/Vis absorption spectra of TMPyP4 and nanotrain@TMPyP4' (The structure of the nanotrain@TMPyP4' was identical to that of nanotrain@TMPyP4 but without any labeling) in aqueous solution.

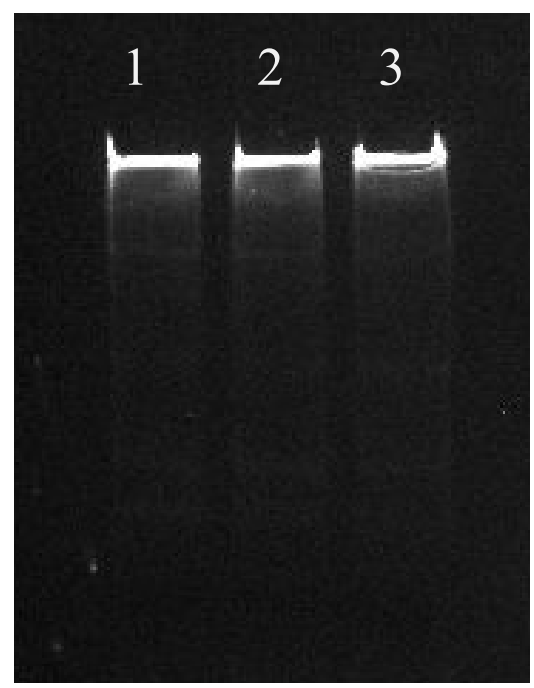

Figure S5. SYBR Green-stained 12\% polyacrylamide gel electrophoresis analysis the stability of nanotrain in water (lane 1), PBS ( $\mathrm{pH}=7.4$, lane 2) and DMEM containing $10 \%$ FBS (lane 3 ) at $37{ }^{\circ} \mathrm{C}$ for $24 \mathrm{~h}$. 


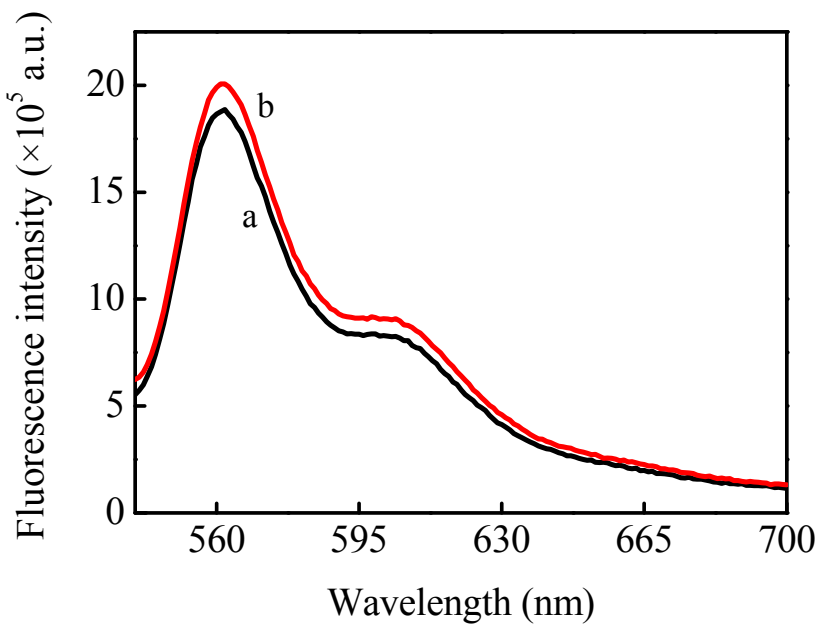

Figure S6. Fluorescence emission spectra of UB-nanotrain before (a) and after (b) reaction with $100 \mu \mathrm{g} \cdot \mathrm{mL}^{-1}$ of liver microsomes and NADPH $(50 \mu \mathrm{M})$ at $37{ }^{\circ} \mathrm{C}$ for 4 h under $1 \% \mathrm{O}_{2}$ (nitrogen gas was bubbled through the reaction mixture mimicked hypoxia).
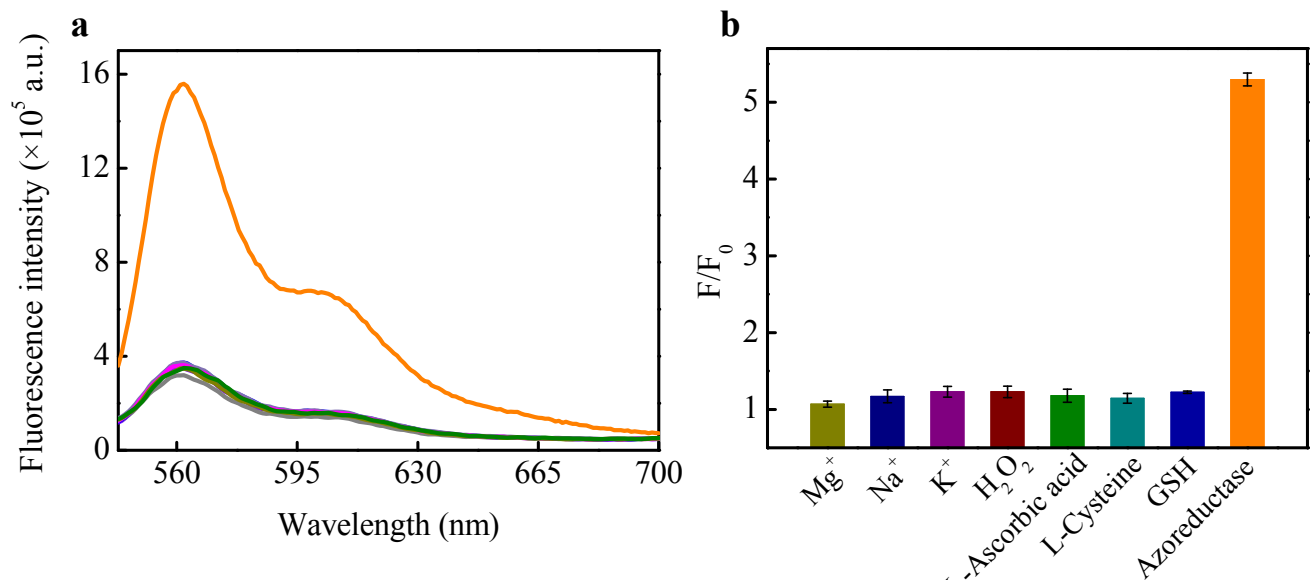

Figure S7. (a) Fluorescence emission spectra of nanotrain in the presence of NADPH $(50 \mu \mathrm{M})$ to various species: $\mathrm{MgCl}_{2}(10 \mathrm{mM}), \mathrm{NaCl}(10 \mathrm{mM}), \mathrm{KCl}(10 \mathrm{mM}), \mathrm{H}_{2} \mathrm{O}_{2}(1$ $\mathrm{mM})$, L-Ascorbic acid (1 mM), L-Cysteine (10 mM), GSH (10 mM) and azoreductase (rat liver microsomes, $\left.100 \mu \mathrm{g} \cdot \mathrm{mL}^{-1}\right)$. (b) Fluorescence enhancement $\left(\mathrm{F} / \mathrm{F}_{0}\right.$, where $\mathrm{F}$ and $\mathrm{F}_{0}$ represents the $\mathrm{Cy} 3$ fluorescence intensity of nanotrain after and before addition 
of various biological species) of the nanotrain upon incubated with different biological species. Error bars represent standard deviations from three repeated experiments.
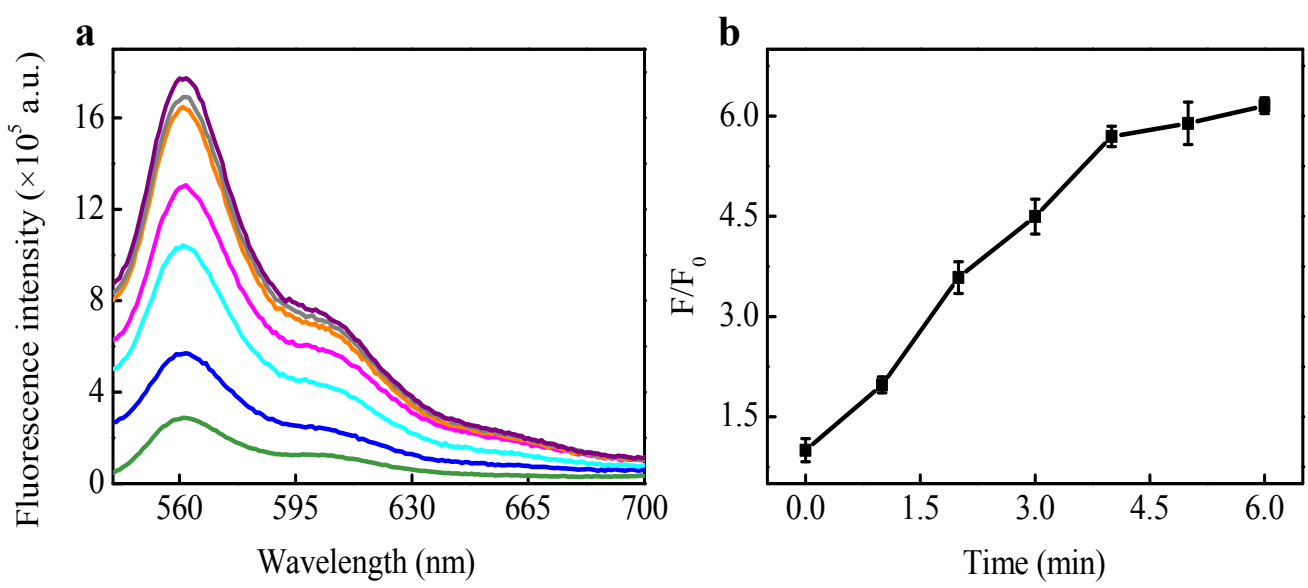

Figure S8. (a) Fluorescence emission spectra of nanotrain upon addition of liver microsomes $\left(100 \mu \mathrm{g} \cdot \mathrm{mL}^{-1}\right)$ and NADPH $(50 \mu \mathrm{M})$ at $37^{\circ} \mathrm{C}$ for different incubation time under $1 \% \mathrm{O}_{2}$. (b) Fluorescence enhancement $\left(\mathrm{F} / \mathrm{F}_{0}\right.$, where $\mathrm{F}$ and $\mathrm{F}_{0}$ represents the fluorescence intensity of $\mathrm{Cy} 3$ after and before incubated with liver microsomes and NADPH for different time) of nanotrain upon incubated with liver microsomes with aid of NADPH $(50 \mu \mathrm{M})$ for different time under $1 \% \mathrm{O}_{2}$. All $1 \% \mathrm{O}_{2}$ conditions were mimicked by bubbling nitrogen in the reaction mixture. Error bars represent standard deviations from three repeated experiments. 

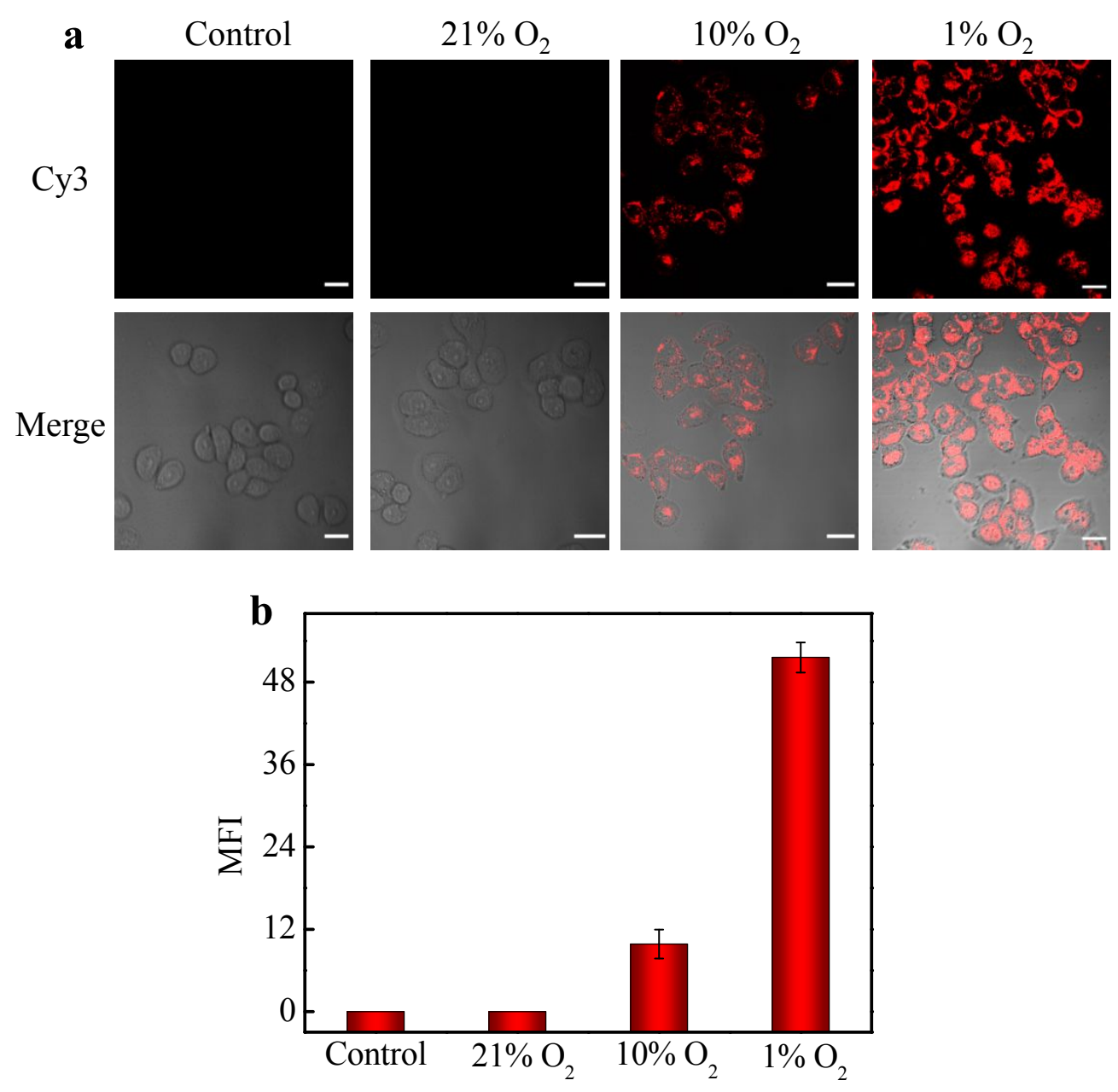

Figure S9. (a) Confocal fluorescence images of MCF-7 cells upon incubated with nanotrain $(50 \mathrm{nM})$ at $37^{\circ} \mathrm{C}$ for $4 \mathrm{~h}$ under different $\mathrm{O}_{2}$ concentrations $(21 \%, 10 \%$ and $1 \% \mathrm{O}_{2}$ ). Scale bar: $20 \mu \mathrm{m}$. (b) The corresponding mean fluorescence intensity (MFI) of $\mathrm{Cy} 3$ for each group in (a). Error bars represent standard deviations from three repeated experiments. 


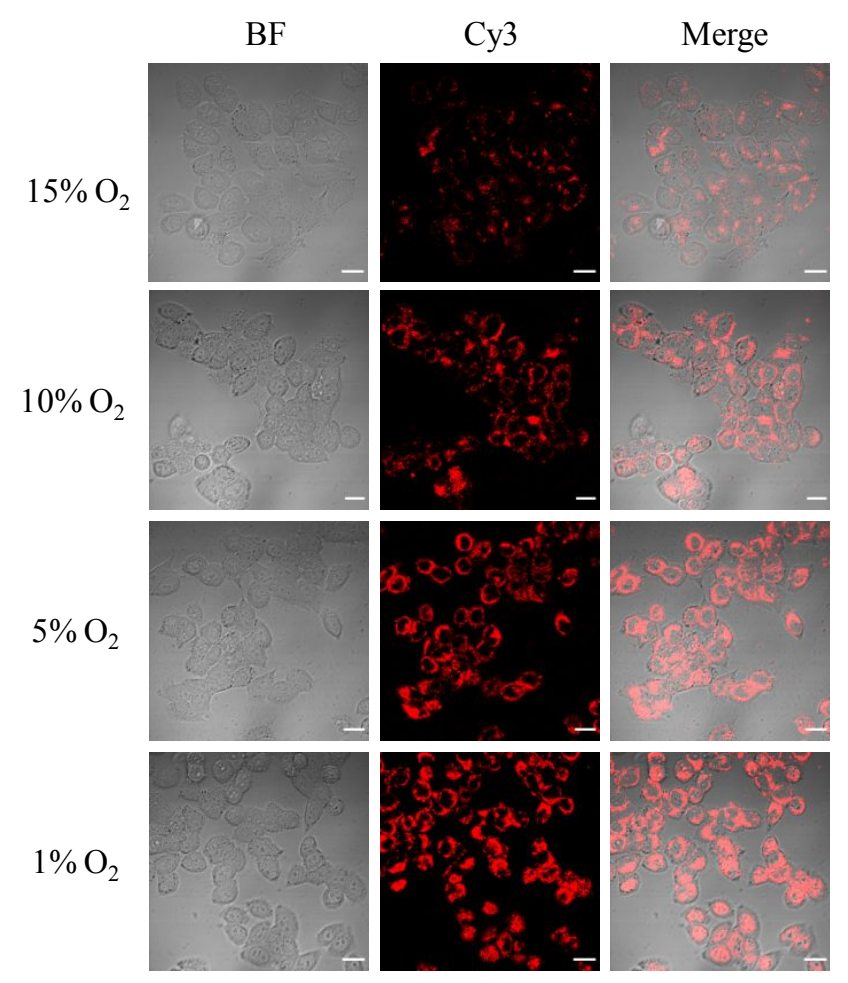

Figure S10. Confocal fluorescence images of MCF-7 cells under different hypoxia conditions $\left(15 \%, 10 \%, 5 \%\right.$, and $\left.1 \% \mathrm{O}_{2}\right)$ upon incubated with nanotrain $(50 \mathrm{nM})$ at $37^{\circ} \mathrm{C}$ for $4 \mathrm{~h}$. Scale bar: $20 \mu \mathrm{m}$. 


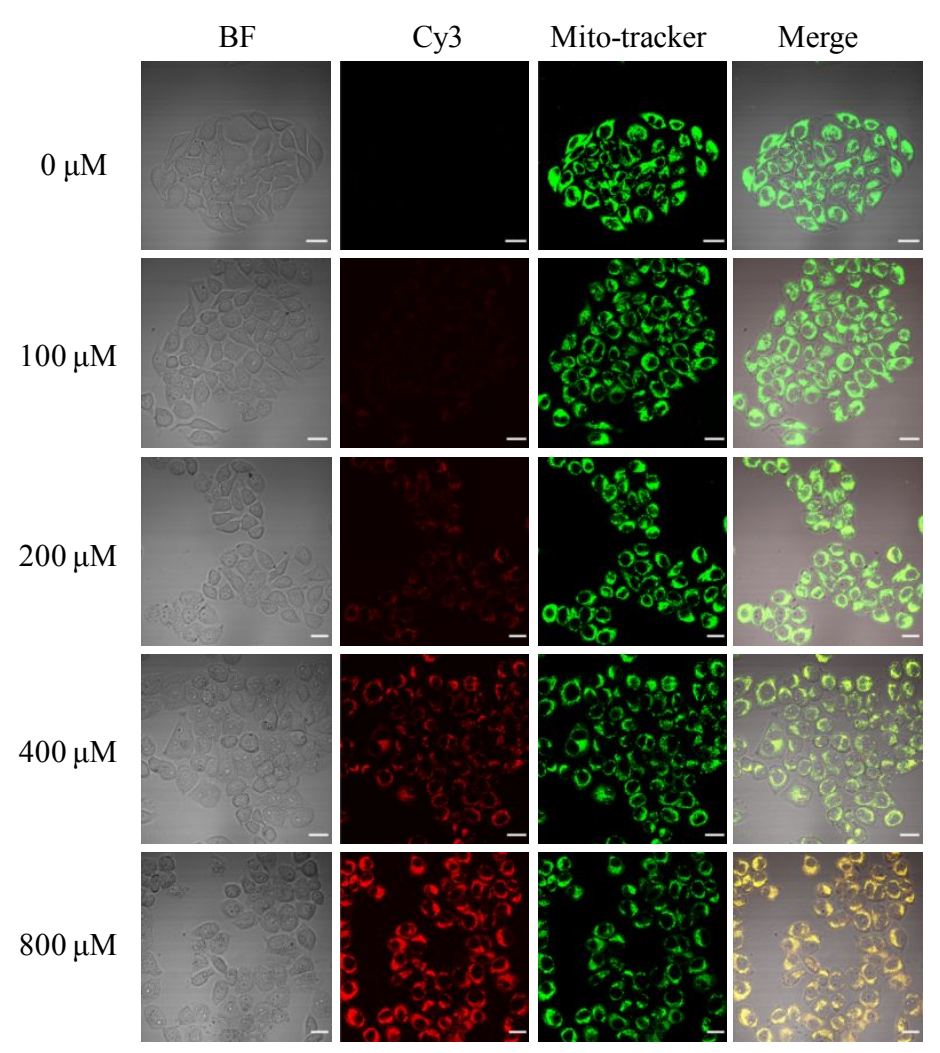

Figure S11. Confocal fluorescence images of the nanotrain-incubated MCF-7 cells upon treatment with different concentrations of $\mathrm{CoCl}_{2}$ for $24 \mathrm{~h}$ under normoxic conditions. Scale bar: $20 \mu \mathrm{m}$. 


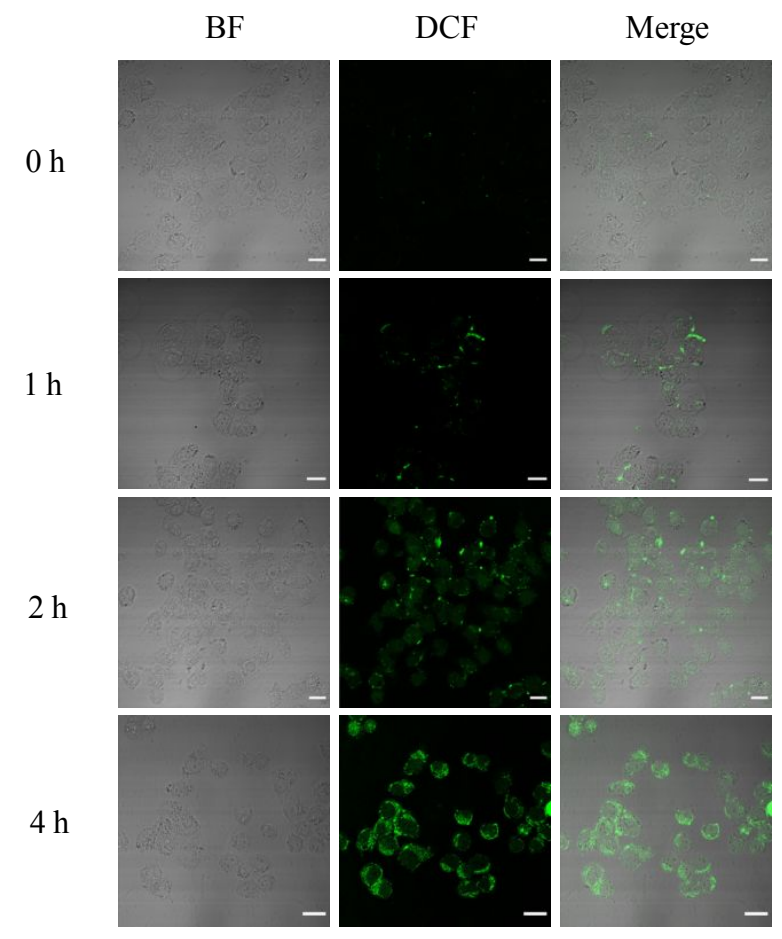

Figure S12. Confocal fluorescence images of $\mathrm{MCF}-7$ cells incubated with nanotrain@TMPyP4 for different time $(0,1,2,4 \mathrm{~h})$ under $10 \% \mathrm{O}_{2}$ condition, and then treated with DCFH-DA following by light irradiation. For the cells required light irradiation, a $660 \mathrm{~nm}$ light at a density of $2.2 \mathrm{~mW} \cdot \mathrm{cm}^{-2}$ was applied for $30 \mathrm{~min}$. Scale bar: $20 \mu \mathrm{m}$. 


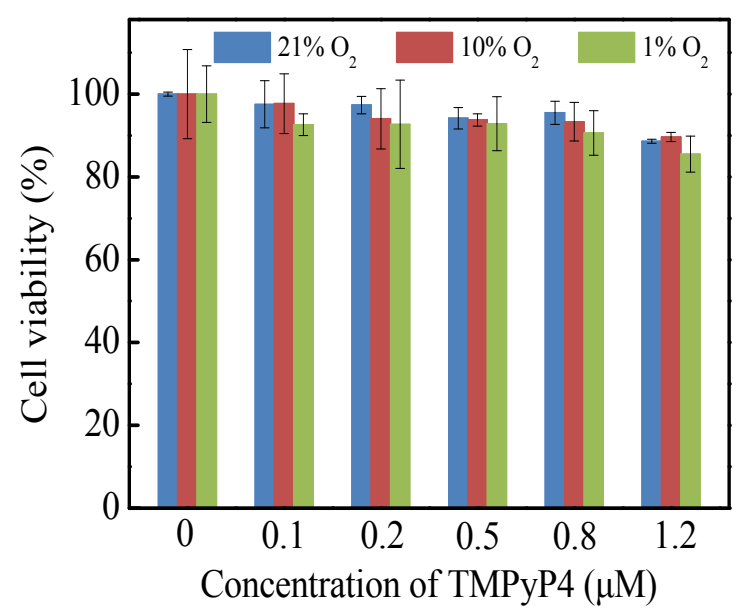

Figure S13. Cell viability of MCF-7 cells upon treated with nanotrain@TMPyP4 for $4 \mathrm{~h}$ under different $\mathrm{O}_{2}$ concentrations $\left(21 \%, 10 \%\right.$ and $\left.1 \% \mathrm{O}_{2}\right)$ without light irradiation. The concentration of nanotrain@TMPyP4 was defined by TMPyP4. Error bars represent standard deviations from three repeated experiments.

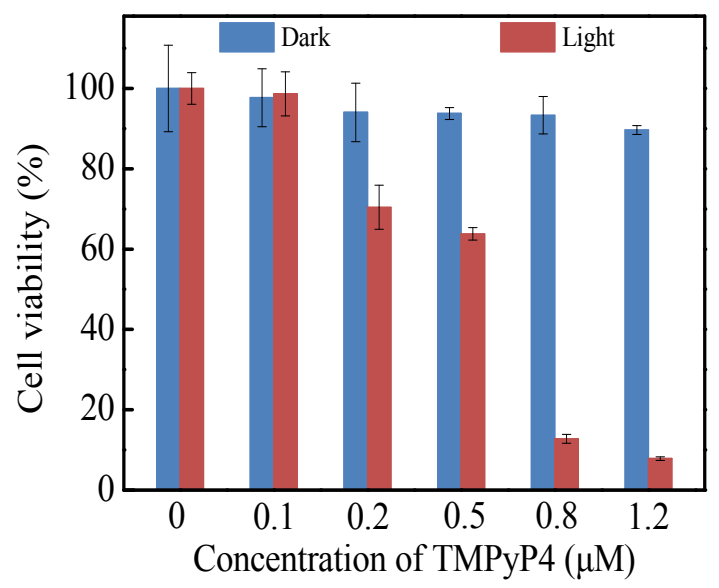

Figure S14. Cell viability of MCF-7 cells upon treated with nanotrain@TMPyP4 under dark (without light irradiation) and light (irradiation with $660 \mathrm{~nm}$ light) condition under $10 \% \mathrm{O}_{2}$. The concentration of nanotrain@TMPyP4 was defined by TMPyP4. Error bars represent standard deviations from three repeated experiments. 


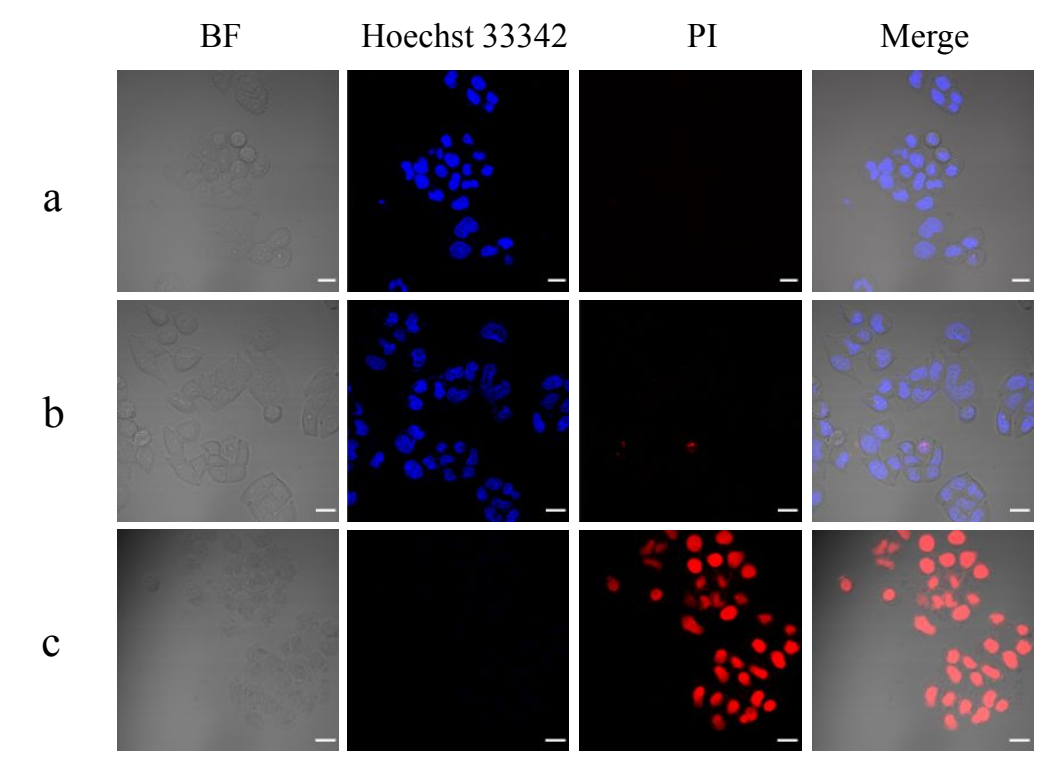

Figure S15. Confocal fluorescence imaging of Hoechest 33342 and PI stained MCF-7 cells which pre-treated with nanotrain@TMPyP4 under different conditions. MCF-7 cells incubated with culture medium (without addition of nanotrain@TMPyP4) under $10 \% \quad \mathrm{O}_{2}$ following by light irradiation (a); MCF-7 cells incubated with nanotrain@TMPyP4 under 21\% $\mathrm{O}_{2}$ following by light irradiation (b); MCF-7 cells treated with and nanotrain@TMPyP4 under 10\% $\mathrm{O}_{2}$ following by light irradiation (c). For the cells required light irradiation, a $660 \mathrm{~nm}$ light at a density of $2.2 \mathrm{~mW} \cdot \mathrm{cm}^{-2}$ was applied for $30 \mathrm{~min}$. Scale bar: $20 \mu \mathrm{m}$. 


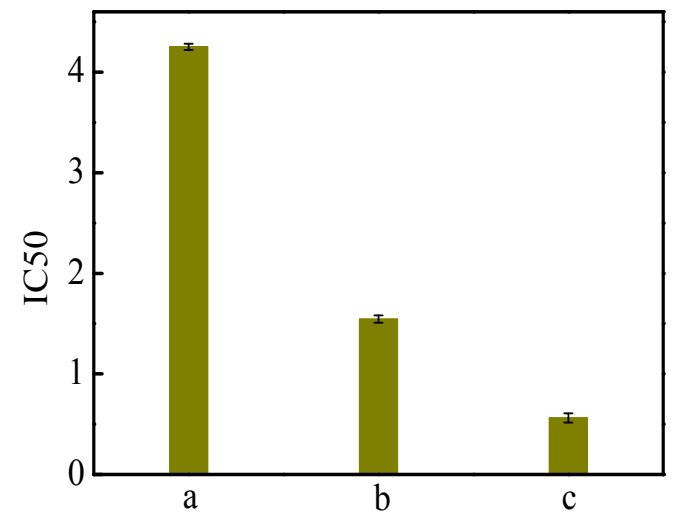

Figure S16. The inhibition concentration (half-maximal inhibitory concentration of a substance, IC50) of TMPyP4-only (a), UC-nanotrain@TMPyP4 (b) and nanotrain@TMPyP4 (c) for MCF-7 cells under 10\% $\mathrm{O}_{2}$ condition. Error bars represent standard deviations from three repeated experiments. 


\section{REFERENCES}

(1) Ge, J.; Lan, M.; Zhou, B.; Liu, W.; Guo, L.; Wang, H.; Jia, Q.; Niu, G.; Huang, X.; Zhou, H.; Meng, X.; Wang, P.; Lee, C.-S.; Zhang, W.; Han, X. A Graphene Quantum Dot Photodynamic Therapy Agent with High Singlet Oxygen Generation. Nat. Commun. 2014, 5, 4596.

(2) Zhu, H.; Li, J.; Qi, X.; Chen, P.; Pu, K. Oxygenic Hybrid Semiconducting Nanoparticles for Enhanced Photodynamic Therapy. Nano Lett. 2018, 18, 586-594.

(3) Pan, M.; Jiang, Q.; Sun, J.; Xu, Z.; Zhou, Y.; Zhang, L.; Liu, X. Programming DNA Nanoassembly for Enhanced Photodynamic Therapy. Angew. Chem. Int. Ed. 2020, 59, 1897-1905.

(4) Frederiksen, P. K.; McIlroy, S. P.; Nielsen, C. B.; Nikolajsen, L.; Skovsen, E.; Jørgensen, M.; Mikkelsen, K. V.; Ogilby, P. R. Two-Photon Photosensitized Production of Singlet Oxygen in Water. J. Am. Chem. Soc. 2005, 127, 255-269. 